C-A/AP/\#207

May 2005

\title{
JLab SRF Institute (SRFI) Trip 8/31/04 - 9/1/04: Final Report
}

\author{
R. Calaga and G. McIntyre
}

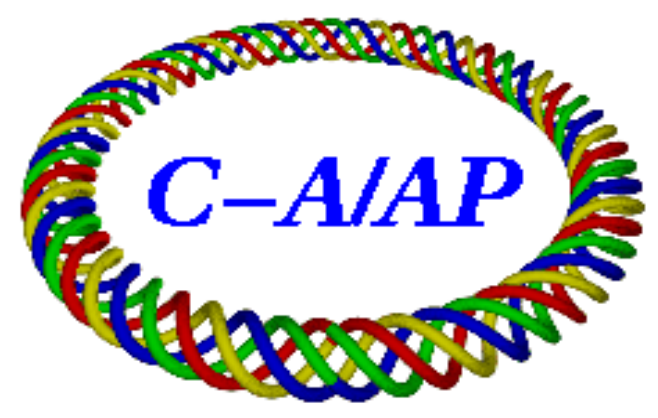

Collider-Accelerator Department Brookhaven National Laboratory

Upton, NY 11973 


\section{JLab SRF Institute (SRFI) Trip 08/31/04 - 09/01/04: Final Report}

Prepared By R. Calaga and G. McIntyre with input from I. Ben-Zvi's trip of 10/01/04

Trip Purpose:

Meetings were held to begin detailing the cleaning and testing steps required for the BNL e-CX SRF cavity. The JLAB facility has many state of the art components including: the chemical cleaning area, the buffered chemical polishing (BCP), high pressure rinse (HPR) facility, bulk hydrogen removal vacuum furnace, the cavity tuning and bead pull testing fixtures, cavity build-up and assembly rail systems and the vertical test area, where cavities with and without helium vessel are tested at superconducting temperature.

The $\mathrm{BCP}$ facility contains a class 100 clean room with a $\mathrm{BCP}$ cabinet, the high pressure rinse cabinet and a cart/rail assembly area. The facility is presently being used to clean and test SNS cavity fabricated by ACCEL Instruments, GmbH, Germany. The cavities are then assembled into a SRF string, and installed in a cryomodule for shipment to the SNS in Oak Ridge, TN.

An initial meeting was held in the office the SRFI's director, Warren Funk. In attendance, during the meeting were: Peter Kneisel, John Mammosser, Larry Turlington, Ed Daly, Joe Preble and Robert Rimmer of JLab and Gary McIntyre and Rama Calaga of BNL.

This report is broken into three sections. First, a chronological list of the tasks that will be requested of the JLab's Superconducting RF Institute (SRFI) in processing the SRF cavity of the BNL e-Cooling Experiment (e-CX). The second section details the processes and discussion about cavity cleaning and testing in general. The final section delineates tasks to be completed and questions answered before the cavity arrives at JLab and who (BNL or JLab) will be responsible for completing or answering the task or question.

\section{Section 1 : JLab Task Chronology}

The meetings' primary motivation was to identify the series of processes and testing to be performed on 5-cell cavity at the JLAB facility. The following is Gary's remembered version of the chronology of the cleaning/ testing / assembly /shipping process.

1. Cavity arrival.

2. CMM measurements / inspection. Cavity vendor AES will collect initial CMM data.

3. RF inspection (AES tuning fixture)

4. Particulate testing of all string components and cleaning as indicated.

5. Degreasing - With their intimate knowledge of both the BCP facility and the needed steps in processing a cavity, JLab will provide a cost estimate on the modification/fabrication of the BCP equipment to be used on the e-CX cavity.

6. Manually rinse cavity after degrease with MicroClean ${ }^{\circledR}$ and dry on cart in class 100 clean room.

7. Initial BCP removal of $200 \mu \mathrm{m}$.

8. HPR and dry in clean room.

9. Bag cavity and crane to furnace.

10. RF tuning of cavity after bake out. 
11. Re-check dimensions (CMM).

12. VTA testing preparation (without He vessel).

a. Degrease.

b. Light BCP $(50 \mu \mathrm{m}$ removed $)$.

c. HPR.

d. Dry on cart in clean room.

e. Assembly on cart

i. Install probe into port

1. Input probe (may be available at JLAB)

2. Sampling or "Pick-up probe"

ii. Seal all ports, including beam line.

1. Requires an isolation valve and 2 burst disk valves.

2. One end of the cavity will have a blank flange and the other the isolation valve and burst disk, second disk plumbed directly to cavity vacuum space.

iii. Leak check system

f. Mount to VTA test stand

g. Cooldown cavity

h. Perform RF testing -with provided instrumentation requests and list of data points needed.

i. Remove from VTA.

j. Disassemble VTA connection from cavity.

k. RF measurements - retuning (final). Tuning must include a "frequency budget" allowing for material to be removed in final BCP / HPR.

1. Cavity is fixtured and the He vessel components are stacked, adjusted and tacked in place.

m. Cavity /He vessel welding completed.

n. Cavity /He vessel prepared for VTA

i. Light BCP

ii. HPR

iii. Dries in clean room.

o. Mount mock tuner.

p. Return to VTA and repeat tests.

q. Depending upon test results, may want use a light BCP/HPR to adjust frequency. (BNL decision point)

r. Cavity /He vessel string assembly begins. (Estimated all assembly tooling required by April 05 .

13. e-CX string assembled in clean room using BNL-supplied/Jlab modified tooling on inner rail system.

14. Mount space frame to outer rail system.

15. Mount Cavity /He vessel assembly to space frame (SF) with Nitronic ${ }^{\circledR}$ rods.

16. Mount "outriggers" to SF.

17. Install string to outrigger hardware.

18. Install string / SF into lower e-CX vacuum vessel.

19. "Construct" vacuum vessel around SF for rigidity. 
20. Move string/vacuum vessel to "strongback". Check to see if vacuum vessel OD matches SNS vessel.

21. Mount to shipping strongback (possible use of SNS strongback must be explored.)

22. Ship via Fedex air-ride van. Only package on the van, direct delivery.

\section{Section 2 Process Details:}

The process steps are as follows:

1. Initial RF testing: Upon receipt from Advanced Energy Systems, Inc., of Medford, New York, the cavity will be inspected mechanically by CMM (Computer Measuring Machine) to measure the cavity's physical dimensions. The cavity will be given a RF inspection and retuned for field flatness, if needed.

2. BCP: The cavity will be given the initial $\mathrm{BCP}$ to remove $200 \mu \mathrm{m}$ from the interior surface and a minimal external $\mathrm{BCP}$ for descaling. The chemistry cabinet dimension are: 65 " $\times 44.5 / 8$ " opening, and 61 " maximum height with base module.

3. Baking: After a water rinse, the cavity is "bagged" and taken to the vacuum furnace for bulk hydrogen removal. This is done under vacuum at $600^{\circ} \mathrm{C}$ for 10 hours.

4. Testing: After removal from the oven the cavity is dimensionally inspected and retuned to guard against frequency change induced by the BCP and bake-out. The AES' tuner may be needed to tune the cavity or plates made to allow the SNS high beta-tuner accept the e-CX cavity.

5. Re-clean and instrument cavity with field probes in preparation for testing in the vertical test dewar.

6. Vertical Test Area (VTA): The following VTA testing was suggested:

a. Q vs. temperature testing

b. Q vs. field testing (Lorentz force detuning coefficient determination). Frequency at different field levels.

i. Fundamental mode

ii. 4 other modes in the bypass band.

c. Pressure sensitivity testing.

d. Penetration depth measurements (if possible).

e. Q disease testing with slow cool down or model with expected cool down rate.

f. BNL goal for accelerating gradient is $15 \mathrm{MV} / \mathrm{m}$ at $\mathrm{Q}$ of $1 \mathrm{X} 10^{10}$. If possible $20 \mathrm{MV} / \mathrm{m}$ with $\mathrm{Q}$ of $2 \times 10^{10}$ is desired. John thought $10^{10}$ might be difficult without additional BCP / HPR runs. Peter also warned that VTA is "under the best of conditions" and the parameters should allow for some margin for the attachment of absorbers, etc. 
7. HOM testing: After each trip to the VTA, done before warm-up, frequency and $\mathrm{Q}$ measurement of HOM modes of interest.

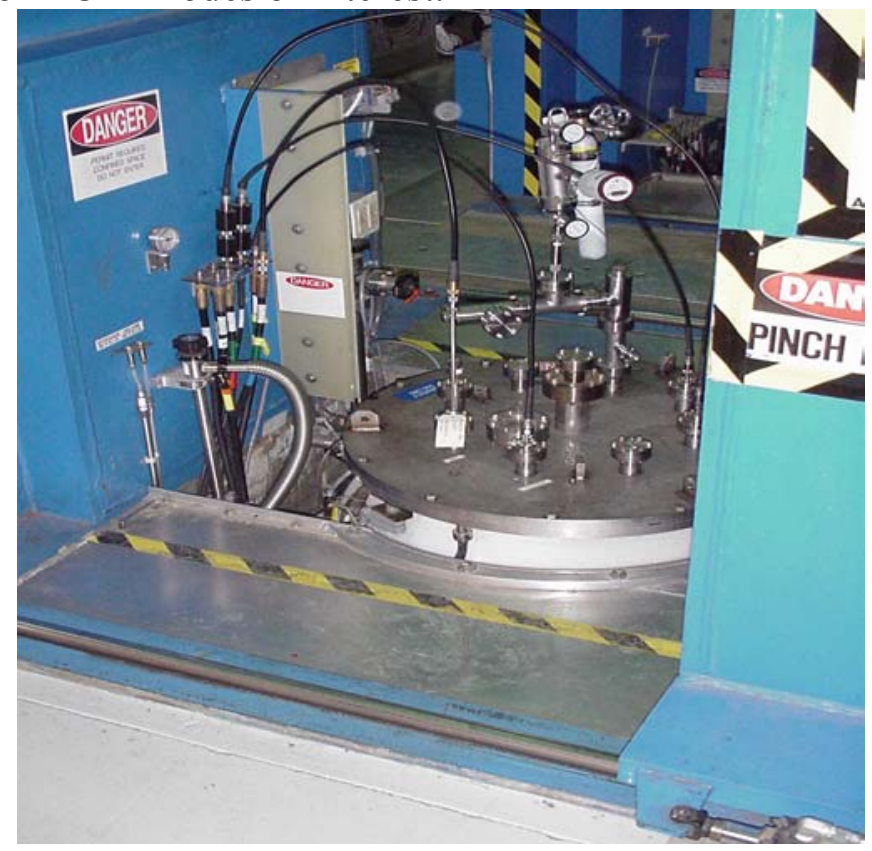

Top of a Vertical Test Dewar

8. Re-BCP: As required, depending upon gradient results and acceptance parameters. The BCP usually removes up to $100 \mu \mathrm{m}$. After BCP a residual resistance of $2-20 \mathrm{n} \Omega$ is expected.

9. High Pressure Rinse (HPR) - The present JLAB HPR system is comprised of a 48 " high, twin nozzle wand with a pressured feed of 1200 psi de-ionized water. The wand starts at the top of the cavity and rotates as it drop 0.2 " / 2 seconds. The e-CX cavity's size prevents using JLAB's set-up. The HPR dimensions: 57"x37.5" opening, 51" height of wand travel distance.

Several HPR options were discussed:

a) Ultra-sonic rinse with flow and agitation - This would be done in the chemical cleaning area and out of the clean room. The cavity would be put into an ultra sonic bath. An agitated flow out DI water would be flowed through the cavity for a yet undetermined period of time. The cavity would 


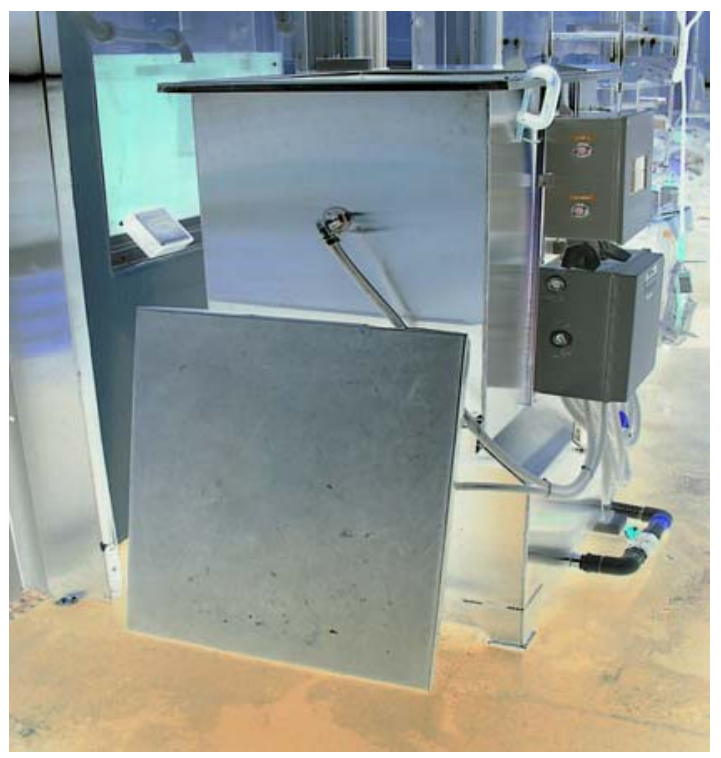

Ultrasonic Cleaner Tank

have to be flipped with process repeated on the formerly unrinsed end. This option requires fixtures which firmly captures the cavity's end flange or iris, etc. and allows hoisting of the cavity by these fixtures. Ultra-sonic system and pumping system exist. Risks - cavity handling and done outside clean room.

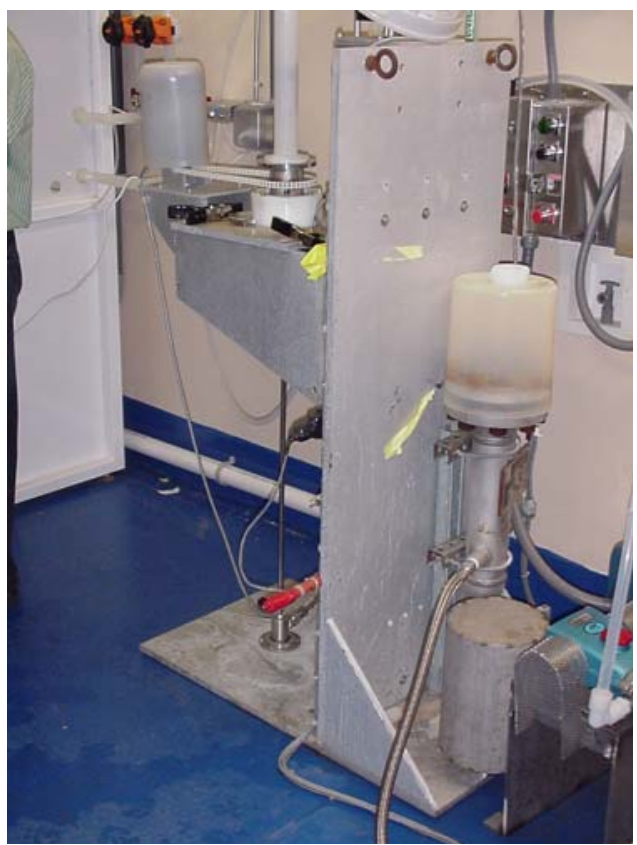

Rotating Wand HPR Stand

b) Rotating Cavity - Put the cavity on a turntable rotate while multi-nozzle wand rinses cavity ID. This option requires turntable, new wand and fixtures to 
support cavity while on table. Pump system exists. (Guestimate: $\$ 12 \mathrm{~K}$ for materials) Risks - Cost, and done outside clean room.

c) Moving Cavity - Using hoist in chemical cleaning area, move cavity up and down over extended wand. This option requires a hoisting fixture which firmly captures the cavity's end flange or iris, etc. and allows steady (small ID tolerance) movement of the cavity.

In all cases the cavity would be dried in the class 100 clean room. The cavity is then sealed, evacuated and back filled to +2 psig argon. Complete: BNL has decided to use option (b). BNL will supply the HPR system. It will be designed to connect to and utilize the JLab high pressure DI water system.

10. He Vessel Assembly: The cavity is mounted in welding fixture. It may be possible to use the SNS fixture if bolt-on bands can be made bringing the vessel OD out to 24" from 20". Cavity is positioned vertically. The components of the He vessel are "stacked and tacked". During this process the sleeves designed into the vessel are used to achieve the correct length, thus avoiding a frequency shifting impact on the tested and tuned cavity. The cavity/vessel is leak checked to $2 \mathrm{X} 10^{-10}$ Torr- $\mathrm{L}(\mathrm{He}) / \mathrm{sec}$. The cavity / He vessel welding should be done in steps with frequency testing done between steps to minimize frequency change use the weld distortion modify frequency if a series of welds produces a negative shift.

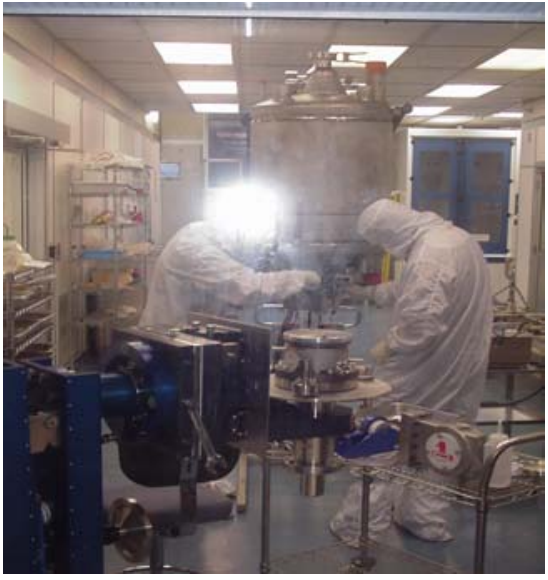

Class 100 Cleanroom Area

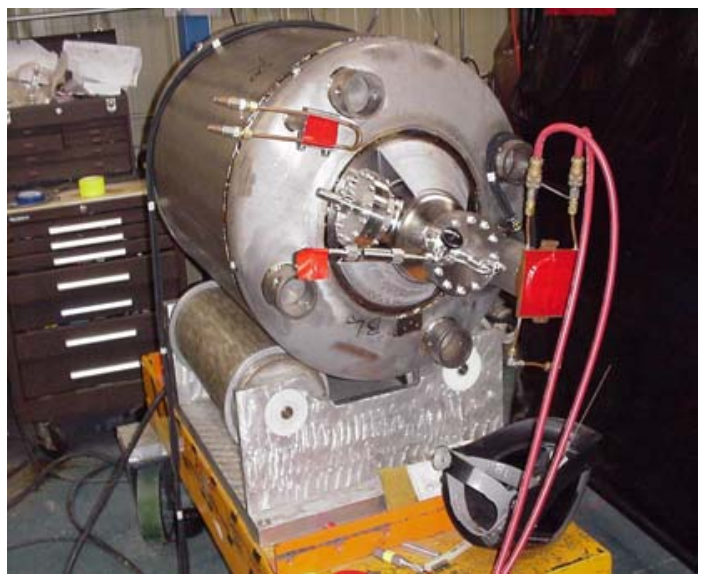

He Vessel's Rolling Weld Fixture

11. Testing: Open the cavity in the clean room and clean string components. May want to do one last field flatness test. Cavity can be re-tuned from outside the $\mathrm{He}$ vessel, but it is ugly and painful. Suggested to put mounting provisions on the $\mathrm{OD} /$ heads of He vessel for easy assembly fixtures (e.g., BCP, mock tuner plate) to cavity.

\section{Additional Procedures:}

a) Particulate testing: All components to be assembled into the cavity string must be tested at JLAB prior to installation. Ask ACCEL for cleaning procedure for the ferrite absorbers. Request their acceptable particulate levels and acceptance testing results for these components. It should be assumed that 
components assembled into the string will have to be cleaned / re-cleaned at JLAB. John requested a list of all components, their constituent materials, cleaning steps done / recommended and any handling requirements.

b) FPC Processing: John asked who will qualify/process/commission the fundamental mode coupler FPC to reach the required power rating. It must be decided who will take responsibility for this component's preparation. John and Peter warned about extensive labor costs in testing and conditioning the FPC. It is an involved process taking 1 week to inspect, leak check, test for blistering and install instrumentation and one week to test. JLAB presently has the equipment to do this testing, but only up to $20 \mathrm{~kW}$ and only until October 2004 when the equipment will be shipped to SNS. SNS contact is Ricky Campisi, formerly of JLAB. There was evidence of the coppercladding on the FPC (from Toshiba) blistered after the required baking. The post-baking step of a HPR is important in determining if the copper plate is still adherent.

c) Alignment: John asked which components in the string were critical for string/beam alignment. What is the tolerance of the perpendicularity of the cavity centerline to the cavity's end flanges? This requirement dictates the alignment of all the other components hung off the cavity/He vessel. John will find the tolerance required by the SNS drawings. Ed explained that the single bellows, between the cavity and the cryomodule weld flange is the only alignment allowance in the string. He suggested considering a second bellows to effect more margin aligning cavity.

d) HOM Couplers: Spoke briefly about HOM coupler and the possibility of not having any on the actual cavity.

e) Weight Check: Re-check / verify cavity and He vessel weights. SNS equipment is rated for a $250 \mathrm{lbs}$ maximum. Send John and Peter drawing package showing actual cavity dimensions. They will verify cabinet / cavity compatibility and lift-cart capacity.

f) Field Mapping: e-CX test area must be mapped for magnetic fields, vertical and horizontal. Rule of thumb: $0.25-0.33 \mathrm{n} \Omega / 0.001$ gauss. Can have a negative effect of cavity performance.

g) Cavity Adapter: Moving equipment for BCP work and assembly needs to lift and rotate cavity and cavity with He vessel. e-CX cavity's Ø20" may allow for use of the SNS lift-carts if it's plausible to create adapter ring to take the 20 " OD to a 24 OD. The Swedish-made lift-carts (Back-Tech 600) cost approximately $\$ 20 \mathrm{~K}$ each. Once JLAB has the final cavity / He vessel weights a determination will be made if these carts can be used on the e-CX cavity. Need to develop cavity flange covers to be removed and re-apply to protect cavity during processing steps. Must examine stress and possible distortion caused by the loading of the cavity flanges / iris etc. by any fixtures. 


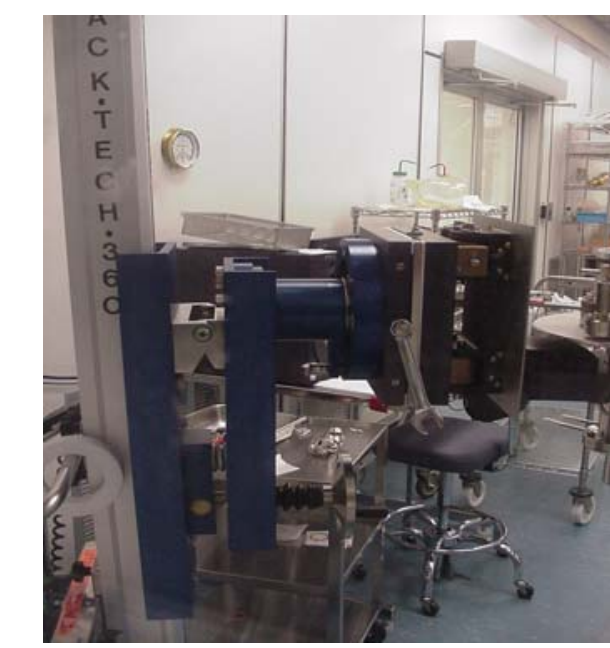

Back-Tech Liftcar in Cleanroom

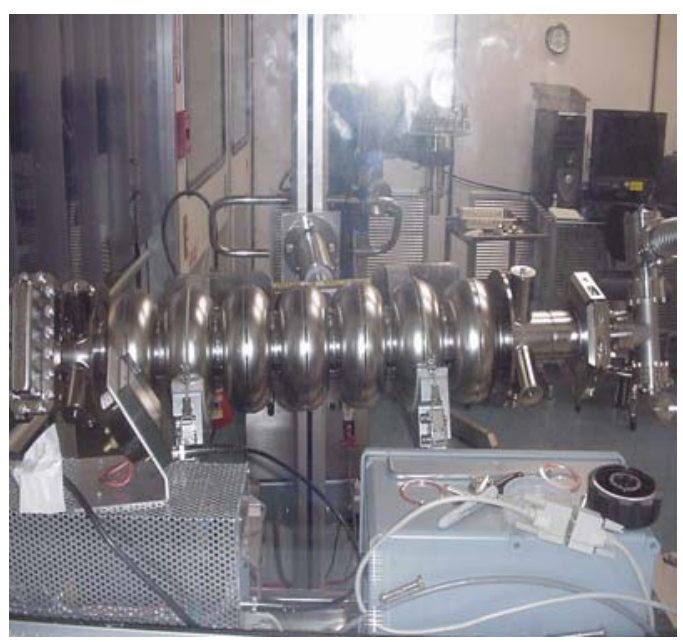

Field Flattening Tuning Fixture

h) Seals: Peter suggested using indium seals in the VTA. Indium is readily available and will save money in seals and the fabrication of expensive flanges and other hardware. Indium sealing may precipitate additional cleaning due to seal residue finding its way into the cavity. Ed and John suggested using actual AlMg3 (with $0.5 \% \mathrm{Si}$ ) in the VTA to gain experience in the use of these gaskets. Due to the small number of seal needed, the expense and the limited time available it was suggested that BNL fabricate their own AlMg3 seals. The JLAB/SNS vendor is German, but none of attendees knew the vendors name. In either case, Nb55TI blank-off flanges will be needed.

\section{Shipping of Cavity String:}

Gary spoke to Mark Wiseman (x7289) seeking advice for shipping of string assembly. Mark was responsible for the shipping of the SNS cryomodules. He said that since the tuner would not be in place the mock-tuner plate should be installed to stabilize the cavity/He vessel bellows. Mark also suggested the plausibility of using the SNS shipping strongback / pallet by mounting the lower and upper e-CX cryomodule vacuum vessel sections to stiffen the space frame during shipping.

Mark said the maximum g-force seen in any direction during shipping was $0.7 \mathrm{~g}$. The system was design for $5 \mathrm{~g}$. The SNS cryomodule is cradled on the bottom with a neoprene "bushing" on the semi-circular receiver. The strongback is separated from the shipping pallet by stiff SST cable ring, which provide cushion while delivering resistance from large displacements in any direction.

\section{Tooling:}

Gary spoke to Ed Daly about design of tooling. Ed was shown the first pass of possible tooling based on present JLab/SNS clean room SRF string assembly tooling.

Ed stated that once the cavity/vessel string clean room assembly is complete, the string is sitting on an inner/upper set of rails. The space frame then rolls over the string using an outer/lower set of rails. The same concept can be used for the e-CX cavity but provision must be made in the SF to accept the supports that emanate from the lower rails. Ed 
suggested using a standard upper cart configuration and fabricating a bridge piece that can support the valve and the tapered sections. A tab, tapped holes in a block welded on or other type receiver, will have to be added to the tapered pieces to do this. Will need multiple ways support valve in addition to present tapped holes in the side of the valve body.

If Jim Rank would like him to review the tuner assembly, Ed offered to sit down with Jim and go over it together.

\section{Tasks to be completed prior to cavity delivery at JLab:}

\section{JLab Tasks}

1) Vertical test dewar fixture's load capacity may be exceeded by the e-CX cavity. $\mathrm{JLab}$ will load test the fixture once the cavity/vessel weight data is received.

2) From cavity/He vessel weight estimate from BNL determine cabinet / cavity compatibility and lift-cart capacity with e-CX cavity.

3) Provide a cost estimate of modifying existing cleanroom/BCP equipment to accommodate the e-CX cavity and He vessel, including the possibility of fabricating bolt-on bands that bring the He vessel OD out from 20 " to 24 " and then using the SNS He vessel welding fixture.

4) JLAB will check the length of vacuum furnace. Complete: useable length of furnace is 65 inches.

5) Find the tolerance required by the SNS drawings for perpendicularity of the cavity centerline to the cavity's end flanges.

6) Investigate possibility of providing the sampling or "pick-up" probe. Complete: JlLab can provide the pick-up probe.

7) Verify input probe for VTA testing is available from JLAB. Complete: probe is available.

\section{BNL Tasks}

1) Obtain permission to use the AES' tuner at JLab on the e-CX cavity. Complete: permission obtained.

2) Determine cavity's actual surface area and volume to perform accurate cavity weight and acid weight calculations. Send John and Peter a drawing package shown cavity dimension details, from which they will determine cabinet / cavity compatibility and lift-cart capacity.

3) Design and fabrication of fixtures needed for HPR option chosen.

4) Determine what kind of cleaning is required for $\mathrm{Nb}-\mathrm{Cu} / \mathrm{SS}$ transition.

5) Estimate frequency change due to chemical cleaning.

6) Course of action if final frequency is wrong after He vessel welding.

7) It was requested that a simplified version of the e-CX cavity / ERL schedule be sent to Warren Funk and Peter Kneisel.

8) Blank-offs for cavity flanges, made from of $\mathrm{Nb} 55 \mathrm{Ti}$, are required for VTA testing.

9) What is the cavity's flange material and are they welded or brazed? Complete: The flanges are made from $\mathrm{Nb55Ti}$ and welded to the cavity.

10) Produce waveguide mock-ups for use in the VTA. 
11) Weld additional mounting provisions on the $\mathrm{OD} /$ heads of He vessel for easy assembly fixtures (e.g., BCP, mock tuner plate) to cavity.

12) Develop a list of all components to be assembled into the string, their constituent materials, cleaning steps done / recommended and any handling requirements.

13) Determine which components in the string are critical for string/beam alignment. What is the tolerance of the perpendicularity of the cavity centerline to the cavity's end flanges?

14) Consider a second bellows, between the cavity and the cryomodule weld flange, to effect more margin in aligning the cavity.

15) Determine the total length of the FPC.

16) Borrow a spare SNS coupler from JLab to allow processing of the FPC by R. Campisi of ORNL

17) Design and supply easy-to-remove/replace covers for all flanges on cavity and string components.

18) Examine stress and possible distortion caused by the loading of the cavity flanges / iris, etc. by any fixtures.

19) Fabricate limited number of $\mathrm{AlMg}(0.5 \% \mathrm{Si})$ seals from bar stock for each flange type on cavity, run seal tests and supply to JLab for testing.

20) Provide instrumentation requests and full list of data point needed.

21) Provide isolation valve (1) and burst disks (2) for ends of cavity beamline.

22) Fabricate the components designed by JLab for adapting to JLab cleanroom equipment.

23) Check to see if e-CX vacuum vessel OD matches SNS vessel and explore possibility of using SNS shipping fixture. Complete: Both vessels are 42" OD.

24) Ask Mark about protection of FPC stem during shipping. 